\title{
Communication
}

\section{USP2a positively regulates TCR-induced NF-KB activation by bridging MALT1-TRAF6}

\author{
Yi Li, Xiao He, Shuai Wang, Hong-Bing Shu, Yu Liu \\ College of Life Sciences, Wuhan University, Wuhan 430072, China \\ $\triangle$ Correspondence: yuliu@whu.edu.cn \\ Received November 29, 2012 Accepted December 4, 2012
}

\begin{abstract}
The paracaspase MALT1 is essential for the activation of NF-KB in response to T cell receptor (TCR) stimulation. It recruits downstream TRAF6 and activates the E3 ligase activity of TRAF6 to polyubiquitinate several targets, which ultimately leads to NF-KB activation. Here we identified ubiquitin-specific protease $2 a$ (USP2a) as a MALT1-associated protein by biochemical affinity purification. Endogenous USP2a constitutively interacted with TRAF6, but dynamically interacted with MALT1 and CARMA1 in a stimulation-dependent manner. RNA interference (RNAi)-mediated silencing of USP2a attenuated TCR-induced NF-KB activation and production of interleukin-2 (IL-2). In addition, the ubiquitination of MALT1 and TRAF6 were both suppressed by USP2a knockdown. By knockdown and reconstitution assays, we found that USP2a mediated the interaction between MALT1 and TRAF6 in a catalytic activity-dependent manner. Furthermore, USP2a deSUMOylated TRAF6. Our findings implicate that USP2a plays an important role in TCR signaling by deSUMOylating TRAF6 and mediating TRAF6-MALT1 interaction.
\end{abstract}

\section{KEYWORDS USP2a, MALT1, TRAF6, T cell activation, $\mathrm{NF}-\mathrm{KB}$}

\section{INTRODUCTION}

NF-KB is an important transcription factor in inducing expression of gene products mediating immune responses. The activation of NF-KB can be triggered by not only pathogenic structures, multiple cytokines and various stress stimuli, but also antigen recognition by lymphocyte receptor complexes (Ghosh et al., 1998).

Full activation of mature $T$ cell depends on the engage- ment of T cell receptor (TCR) complex and CD28 costimulatory receptor by an antigen-presenting cell (Alberola-lla et al., 1997). TCR/CD28 costimulation induces classical NF-KB signaling, which depends on the activation of the IKB kinase (IKK) complex, composed of two kinase subunits IKKa and $\mathrm{IKK} \beta$, and a regulatory subunit IKKy. IKK complexes catalyze the phosphorylation of the $1 \mathrm{~KB}$ inhibitor $1 \mathrm{~KB} a$, which subsequently undergoes proteasomal-dependent degradation. Cytosolic NF-KB is then released, translocates into the nucleus, and engages $\mathrm{kB}$ enhancer elements of target genes.

In $\mathrm{T}$ cells, three indispensable signal proteins, caspaserecruitment domain membrane-associated guanylate kinase protein 1 (CARMA1), B-cell CLL-lymphoma 10 (BCL10) and mucosa-associated lymphoid tissue lymphoma translocation gene 1 (MALT1) assemble a CARMA1/BCL10/MALT1 (CBM) signalosome, which provides a critical link between TCR/ CD28 proximal signaling events and NF-KB activation (Ruland et al., 2001; Gaide et al., 2002; Pomerantz et al., 2002; Wang et al., 2002; Ruefli-Brasse et al., 2003). Upon TCR stimulation, the scaffold protein CARMA1 is phosphorylated and recruited to the lipid rafts. Activated CARMA1 undergoes a conformational change and recruits constitutively associated BCL10-MALT1-IKK $\beta$ to form so called CARMA1/BCL10/MALT1 (CBM) signalosome. Subsequently, BCL10 is phosphorylated to regulate IKK/NF-KB activation both positively and negatively (Wegener et al., 2006; Thome and Weil, 2007), whereas MALT1 is oligomerized and recruits the ubiquitin E3 ligase tumor necrosis factor receptor-associated factor 6 (TRAF6) (Sun et al., 2004). Recruited TRAF6, activated by oligomerization, catalyzes the Lys63linked polyubiquitination of MALT1 and BCL10, both of which thereby mediate the recruitment of IKKY to the CBM signalosome (Oeckinghaus et al., 2007). Furthermore, TRAF6 catalyzes the bound IKKY, whose ubiquitination is essential for IKK activation (Deng et al., 2000). TRAF6 also targets itself for $\mathrm{K} 63$ polyubiquitination and activates the protein ki- 
kinase transforming growth factor $\beta$-activated kinase 1 (TAK1) and TAK1-binding protein 2 (TAB2), which in turn phosphorylate IKK $\beta$ and subsequently lead to IKK activation (Sun and Chen, 2004). Thus, MALT1 acts as a scaffold protein bridging activated CARMA1/BCL10 with TRAF6, and activates IKK complexes by promoting E3 ligase activity of recruited TRAF6. In addition, as a paracaspase, MALT1 possesses a caspase-like proteolytic activity. Upon TCR costimulation, activated MALT1 cleaves the associated de-ubiquitinating enzyme A20, inhibits A20-dependent de-ubiquitination of IKKY, and thereby promotes maximum TCR-induced NF-KB activation (Coornaert et al., 2008). MALT1 also cleaves BCL10, which promotes integrin-mediated adhesion of activation T cells (Rebeaud et al., 2008).

Because of these critical roles of MALT1 in TCR-induced NF-KB activation, we performed biochemical affinity purification with MALT1 as a bait protein to search potential regulators of MALT1 function. USP2a was identified as a protein specifically associated with MALT1. USP2a positively regulated TCR-induced NF-KB activation and production of IL-2 by mediating the interaction between TRAF6 and MALT1. Our study revealed an important role of USP2a in TCR-signaling.

\section{RESULTS}

\section{Identification of USP2a as a MALT1-interacting protein}

MALT1 plays an essential role in TCR-induced NF-KB activation. For a better understanding of the detailed mechanism of TCR signaling, we purified MALT1-associated proteins by a tandem affinity purification experiment, and identified the eluted proteins by a shotgun mass spectrometry analysis method. By comparing with other unrelated purifications using the same method, we identified several candidates, including USP2a, as potential MALT1-associated proteins. To confirm whether these candidates indeed interact with MALT1, we performed coimmunoprecipitation assays. Among the candidates, USP2a interacted with MALT1 specifically. As shown in Fig. 1A, overexpressed USP2a interacted with MALT1, as well as CARMA1, TRAF6 and TAK1, but not with BCL10. To determine the endogenous association of USP2a with these signal proteins, we stimulated Jurkat $T$ cells with PMA/ionomycin (P/l), then performed coimmunoprecipitation assays. The results showed that USP2a was dynamically associated with endogenous MALT1 and CARMA1 upon P/l stimulation (Fig. 1B). In contrast, USP2a was constitutively associated with TRAF6 in T cells, consistent with our previous study (He et al., 2012).

\section{USP2a mediates TCR-induced T cell NF-KB activation and IL-2 production}

Since USP2a was associated with several key signaling proteins in TCR signaling, we examined whether USP2a participated in T cell activation. To examine the physiological role of USP2a in T cells, we made three RNAi vectors targeting different sites of the human USP2a mRNA, and determined their efficiencies. As shown in Fig. 2A, the \#3 USP2a-RNAi plasmid could markedly inhibit the expression of transiently transfected USP2a, whereas \#1 and \#2 RNAi plasmids had minimal effects. We then chose the \#3 RNAi plasmid for further experiments. Stable Jurkat $T$ cells transfected with USP2a-RNAi were generated by retroviral-mediated transduction. As shown in Fig. $2 \mathrm{~B}$, the level of endogenous USP2a in USP2-RNAi- transfected Jurkat T cells was decreased to less than $10 \%$ of control cells. We then stimulated RNAi-transfected cells by crosslinking CD3 and $\mathrm{CD} 28$ or P/l stimulation. The activation status of NF-KB was then measured by phosphorylation of $\mathrm{IkB \alpha}$. As shown in Fig. 2C, knockdown of USP2a dramatically inhibited the activation of NF-KB as indicated by much weaker phosphorylation of $1 \mathrm{KBa}$, suggesting that endogenous USP2a mediates TCR-triggered NF-KB activation. To further confirm the results, we reconstituted the expression of USP2a in USP2a-knockdown cells by transfecting MIGR-GFP-USP2a into USP2a-knockdown cells and subsequently isolating the reconstituted cells by FACS sorting. By immunoblot analysis, we confirmed the level of USP2a in reconstituted cells was partially recovered (Fig. 2D). Upon stimulation by crosslinking $\mathrm{CD} 3$ and CD28, the repression of phosphorylation of IKBa by USP2a-knockdown was partially rescued by USP2a reconstitution (Fig. 2E). Consistently, IL-2 expression induced by $\mathrm{P} / \mathrm{l}$ stimulation was markedly downregulated in USP2aknockdown cells; whereas USP2a reconstitution partially reversed the inhibition of IL-2 transcription and secretion by USP2a knockdown (Fig. $2 \mathrm{~F}$ and $2 \mathrm{G}$ ). These data suggest that USP2a is important for TCR-triggered NF-KB signaling.

\section{Inhibition of TCR-induced ubiquitination of MALT1 and TRAF6 by USP2a knockdown}

USP2a is reported as an ubiquitin-specific protease cleaving the polyubiquitin chains linked to target proteins. Since the ubiquitination of MALT1 and TRAF6 was critical for processing the TCR-induced NF-kB activation, we next tested whether USP2a played a role in regulating the ubiquitination of MALT1 or/and TRAF6. Stable USP2a-RNAi transfected or control Jurkat T cells were treated with CD3/CD28 crosslinking or $\mathrm{P} / \mathrm{l}$ stimulation. Cell lysates were immunoprecipitated with an antibody against MALT1 or TRAF6 for detecting the endogenous polyubiquitination. The results surprisingly showed that either polyubiquitination of MALT1 or TRAF6 in USP2a-knockdown cells were diminished compared with the controls (Fig. 3). Lys63-linked ubiquitination of MALT1 or TRAF6, critical for signal transduction, were also diminished in USP2a-knockdown cells (Fig. 3). These results suggest that USP2a plays a role in TCR-triggered NF-KB activation independent of its deubiquitination activity under physiological condition. 
A

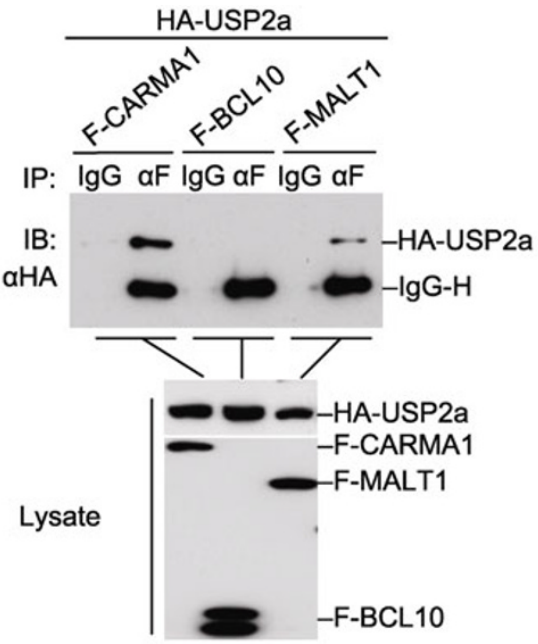

B

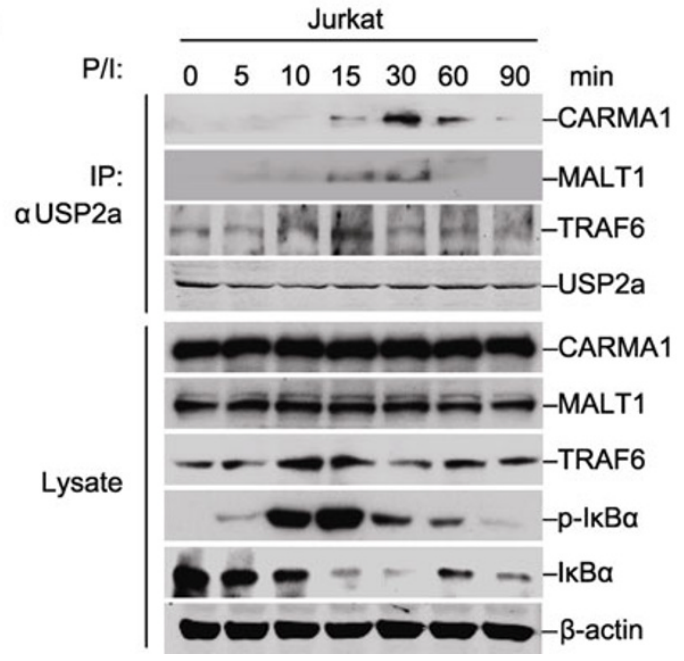

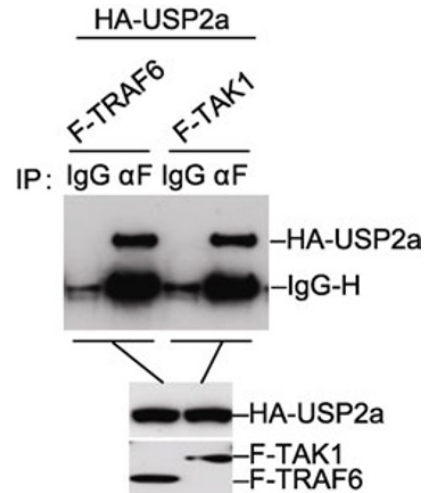

Figure 1. USP2a interacts with CARMA1, MALT1 and TRAF6. (A) HEK293 cells $\left(5 \times 10^{6}\right)$ were transfected with the indicated plasmids (8 $\mu \mathrm{g}$ each). Coimmunoprecipitation (IP) and immunoblot analysis (IB) were performed with the indicated antibodies. $F$, Flag epitope; $\alpha \mathrm{F}$ : anti-Flag antibody; $\alpha \mathrm{HA}$ : anti-HA antibody; IgH, IgG heavy chain. (B) Jurkat T cells $\left(1 \times 10^{8}\right)$ were left untreated or treated with $\mathrm{PMA} / \mathrm{lonomycin}(\mathrm{P} / \mathrm{l}, 1 \mathrm{mmol} / \mathrm{L}$ each) for the indicated times. Coimmunoprecipitation and immunoblots analysis were performed with the indicated antibodies.

\section{Recruitment of TRAF6 to MALT1 by USP2a}

The recruitment of TRAF6 to MALT1 is critical for activating the E3 enzymatic activity of TRAF6 targeting both MALT1 and itself. Because USP2a knockdown reduced the ubiquitination of both MALT1 and TRAF6, we wondered whether USP2a played a role in linking TRAF6 with MALT1. We transfected MALT1 and TRAF6 or BCL10 as a control into USP2a-RNAi or control HEK293 cells, and then performed coimmunoprecipitation experiments. The results showed USP2a knockdown inhibited the association between MALT1 and TRAF6, but had no marked effect on the interactions between MALT1 and BCL10 (Fig. 4A), suggesting that USP2a may act as an adaptor linking the interaction between MALT1 and TRAF6. To confirm this observation, we per- formed endogenous coimmunoprecipitation experiments with USP2a-knockdown Jurkat T cells. Compared with the control cells, USP2a knockdown largely impaired the recruitment of TRAF6 to MALT1 (Fig. 4B).

Because USP2a is a cysteine protease physiologically associated with TRAF6, we next wondered whether the enzymatic activity of USP2a is required for its role in linking TRAF6 to MALT1. We prepared several point mutants of USP2a, in which the enzymatic activities were impaired as reported before (He et al., 2012). TRAF6 and MALT1 were transfected together with USP2a or its point mutant C276A, D357A or H557A in USP2a-knockdown HEK293 cells. The interaction between TRAF6 and MALT1 was then examined by coimmunoprecipitation. The results showed that the re- 
A

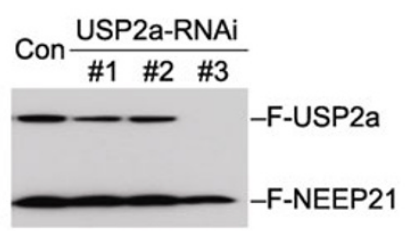

B

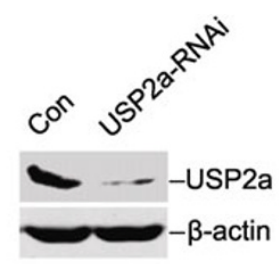

C

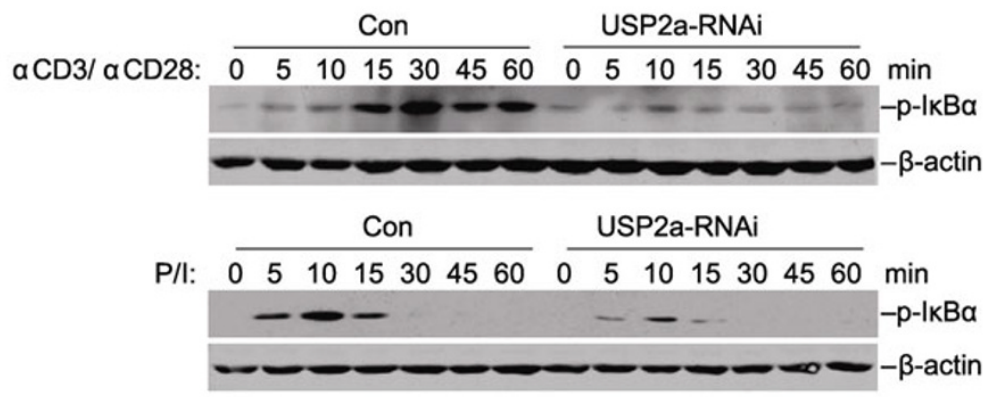

D

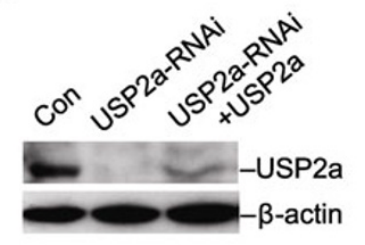

$E$

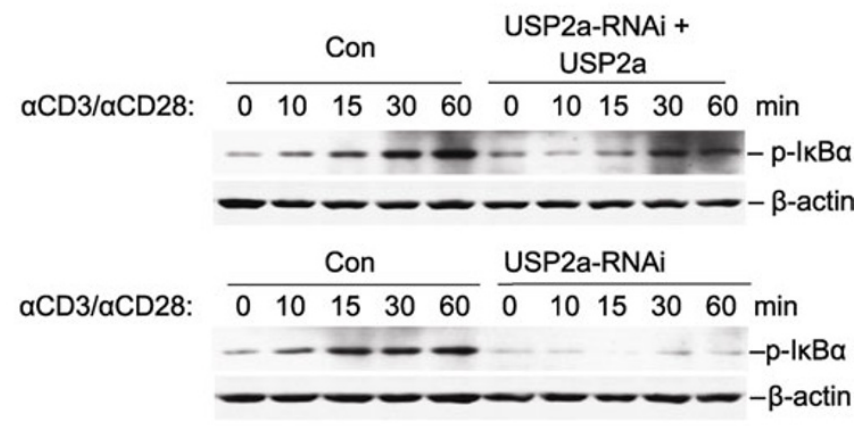

$\mathrm{F}$

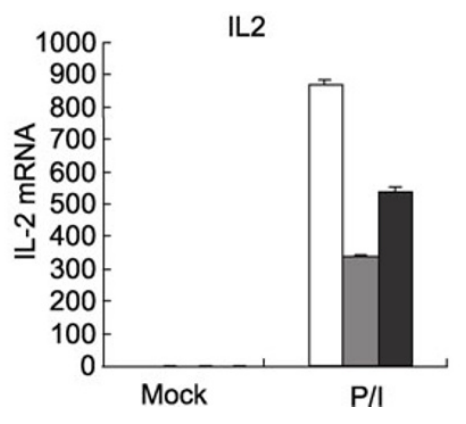

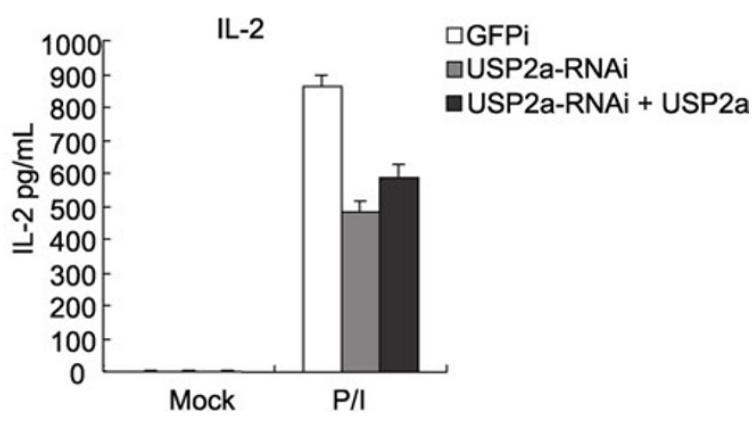

Figure 2. Knockdown of USP2a inhibits TCR-induced NF-KB activation. (A) HEK293 cells $\left(5 \times 10^{5}\right)$ were transfected with Flag-USP2a $(0.2 \mu \mathrm{g})$ and the indicated RNAi plasmids $(1 \mu \mathrm{g})$ together with Flag-NEEP21 $(0.05 \mu \mathrm{g})$ as an internal control. Cell lysates were analyzed by immunoblots with anti-Flag. (B) Jurkat T cells stably transfected with USP2a-RNAi or control RNAi were lyzed and analyzed by immunoblots with the indicated antibodies $\left(6 \times 10^{6}\right.$ cells for each lane). (C) Jurkat T cells stably transfected with USP2a-RNAi or control RNAi were left untreated or treated with CD3/CD28 crosslinking antibodies $(1 \mu \mathrm{g} / \mathrm{mL})$ or P/l $(1 \mathrm{mmol} / \mathrm{L}$ each) for the indicated times. Cell lysates were analyzed by immunoblots with the indicated antibodies. (D) USP2a-knockdown Jurkat T cells were reconstituted with MIGR-GFP-USP2a by retroviral transfection. Seventy-two hours later, reconstituted cells were sorted by FACS. The efficiency of reconstitution was determined by immunoblot with an antibody to USP2a or $\beta$-actin $\left(6 \times 10^{6}\right.$ cells for each lane). (E) USP2a-knockdown Jurkat T cells reconstituted with USP2a or a control vector were stimulated by CD3/CD28 crosslinking antibodies. The cell lysates were analyzed by immunoblots with the indicated antibodies. (F) USP2a-knockdown Jurkat T cells reconstituted with USP2a or a control vector $\left(1 \times 10^{6}\right)$ were stimulated with P/l for 3 hours. The total RNA from the cells was extracted and analyzed by real-time PCR using primers specific for IL-2 genes (left). The culture medium was collected and subjected to ELISA for measuring the production of IL-2 (right). 
A

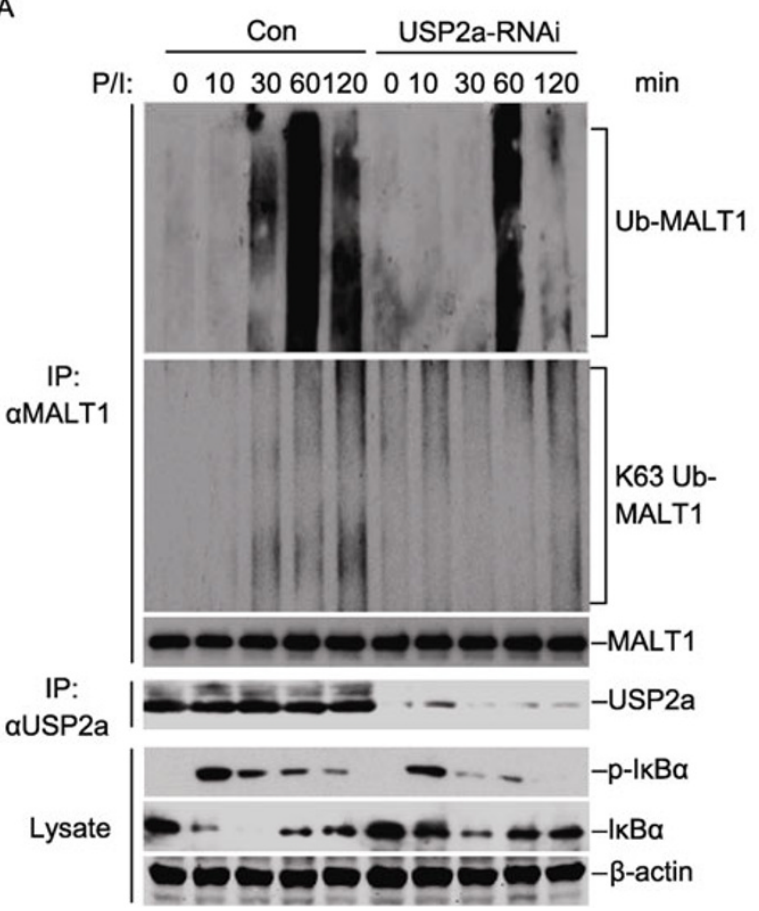

B

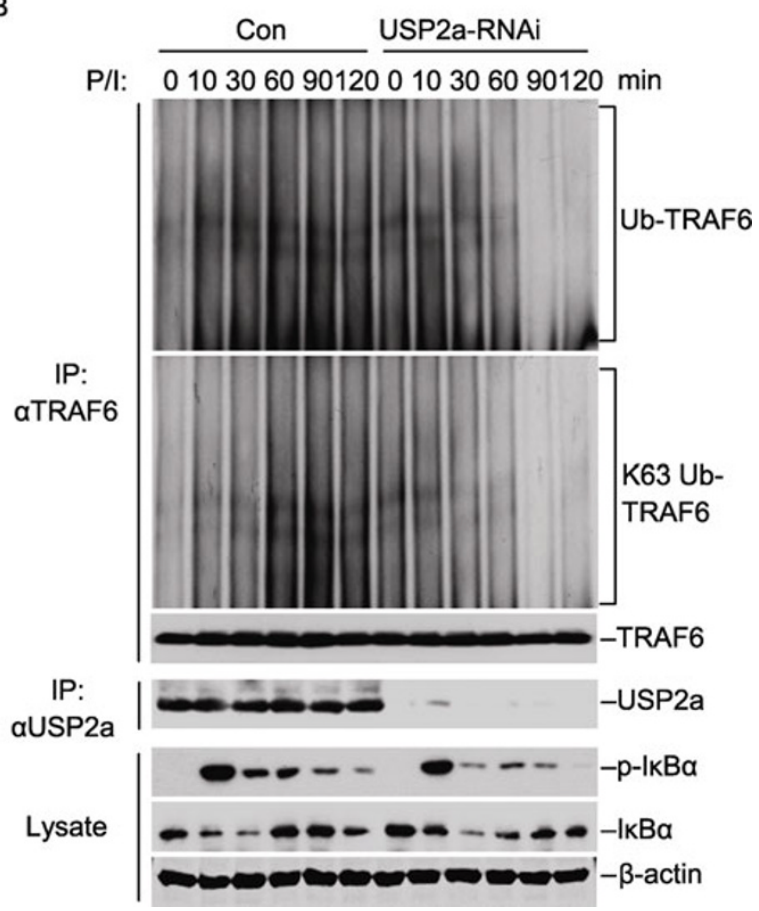

Figure 3. Knockdown of USP2a reduces TCR-induced K63-linked polyubiquitination of endogenous MALT1 and TRAF6. Jurkat T cells $\left(5 \times 10^{7}\right)$ stably transfected with USP2a-RNAi or control RNAi were stimulated with P/l for the indicated times. Cell lysates were coimmunoprecipitated with anti-MALT1 (A) or anti-TRAF6 (B) antibodies. The immunoprecipitates and lysates were analyzed by immunoblots with the indicated antibodies.

constitution of USP2a strengthened TRAF6-MALT1 interaction, further supporting the function of USP2a in recruiting TRAF6 to CBM complex (Fig. 4C). In addition, the reconstitution of point mutants of USP2a, especially D357A and H557A, had less effect on promoting TRAF6-MALT1 interaction than wild-type USP2a did (Fig. 4C). These results suggest that USP2a mediates the interaction between TRAF6 and MALT1, and the enzymatic activity of USP2a is required for its adaptor role.

\section{USP2a mediates the deSUMOylation of TRAF6}

SUMOylation, a post-translational modification of proteins by small ubiquitin-like modifier (SUMO), modulates diverse cellular processes, including transcription, intracellular transport, DNA repair, replication and cell signaling (Hannoun et al., 2010). It has been reported that TRAF6 can be modified by SUMO-1, and thus represses c-Myb-mediated transactivation (Pham et al., 2008). Because the enzymatic activity of USP2a was required for the recruitment of TRAF6 to MALT1, we next determined whether USP2a affects the SUMOylation of TRAF6. In Jurkat T cells, the SUMOylation of TRAF6 was dramatically increased by knockdown of USP2a (Fig. 5A), suggesting that USP2a mediates the removal of the SUMO moiety of TRAF6 under physiological conditions. Furthermore, overexpression of USP2a deSUMOylated overexpressed
TRAF6 in HEK293 cells (Fig. 5B). These results suggest that USP2 mediates the deSUMOylation of TRAF6.

\section{DISCUSSION}

The association of MALT1 and TRAF6 is a critical step linking CBM complex with TAK1-IKK complex in TCR signaling. Upon TCR stimulation, MALT1 is recruited to CARMA1 together with BCL10. Thereafter, MALT1 undergoes oligomerization and recruits TRAF6 through its C-terminal TRAF6 binding sites. The ubiquitin ligase activity of TRAF6 is then activated to mediate K63-linked polyubiquitination of both itself and MALT1, which promoting the signals to downstream NF-KB activation (Sun et al., 2004). Our recent study showed that protein kinase $C$ delta negatively regulates TCR-induced NF-KB activation through interrupting MALT1-TRAF6 interaction (Liu et al., 2012). In this report, we further identify USP2a as an adaptor protein facilitating MALT1-TRAF6 interaction, and therefore promoting NF-KB activation. We provide several lines of evidences in support of our conclusion. First, knockdown of USP2a expression led to the attenuated NF-KB activation and IL-2 production in Jurkat $T$ cells induced by TCR stimuli, whereas the reconstitution of USP2a partially rescued NF-KB activation. These two observations suggest that USP2a is important for TCR signaling. Second, USP2a, constitutively associated with 
A

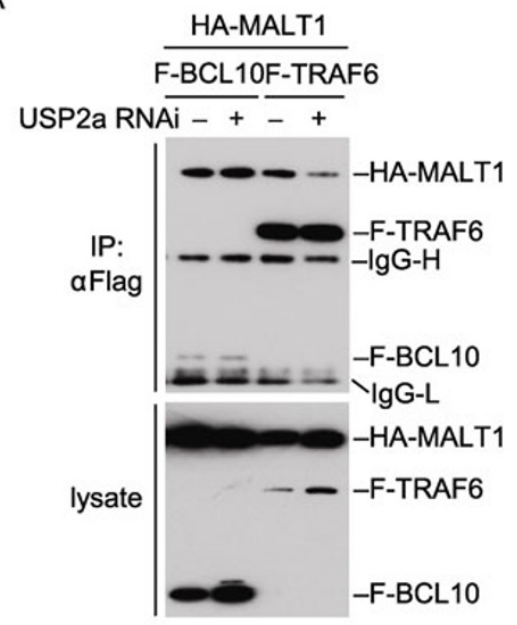

C

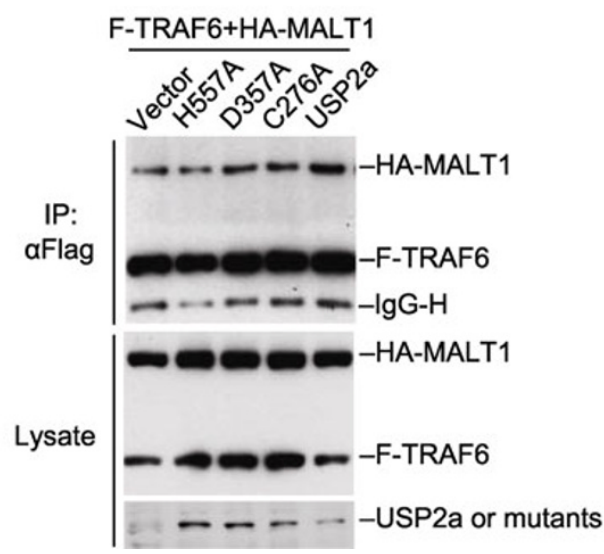

B

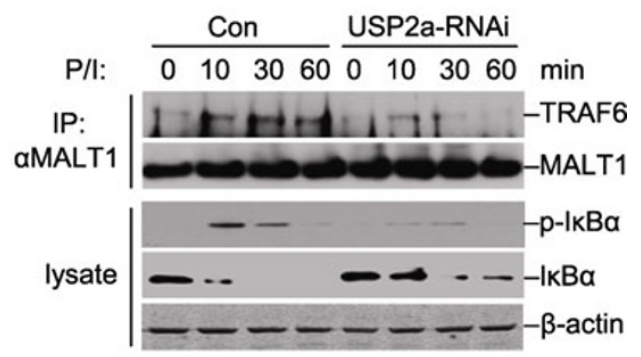

Figure 4. Knockdown of USP2a inhibits TCR-induced association between MALT1 and TRAF6. (A) HEK293 cells stably transfected with control or USP2a-RNAi were transiently transfected with the indicated plasmids (8 $\mu \mathrm{g}$ each). Coimmunoprecipitation and immunoblot analysis were performed with the indicated antibodies. (B) Stable USP2a-knockdown Jurkat T cells or control cells $\left(1 \times 10^{8}\right)$ were stimulated with $\mathrm{P} / \mathrm{l}$ for the indicated times. Immunoprecipitation and immunoblot analysis were performed with the indicated antibodies. (C) Stable USP2a-knockdown HEK293 cells were transfected with Flag-TRAF6, HA-MALT1 and wild-type USP2a, USP2a point mutations or control vector (8 $\mu \mathrm{g}$ each). Coimmunoprecipitation and immunoblot analysis were performed with the indicated antibodies.

ing that USP2a plays a role in TCR signaling independent of its deubiquitination activity under physiological condition. En dogenous USP2a was constitutively associated with TRAF6 and dynamically associated with MALT1 upon the stimulation. The findings by immunoprecipitation assays on both RNAi-transfected and reconstituted cells reveal the role of USP2a in promoting the interaction between TRAF6 and MALT1 as an adaptor.

The findings that the intact enzymatic activity of USP2a is required for its adaptor role triggered us to explore how USP2a regulates the interaction between TRAF6 and MALT1. Our results surprisingly showed that endogenous USP2a deSUMOylated TRAF6. There are only two types of deSUMOylases are reported so far, including the sentrin-specific proteases (SENPs) and deSUMOylating isopeptidase 1 (DeSI-1) (Shin et al., 2012). In the present study, we showed that USP2a, a member of the ubiquitin-specific protease family, could mediate the deSUMOylation of TRAF6. This finding suggests that certain deubiquitinating enzymes may also have deSUMOylating activity.

SUMOylation of target proteins can either provide surfaces for protein interactions, or prevent or lossen protein-protein interaction because of their bulkiness (Bergink 


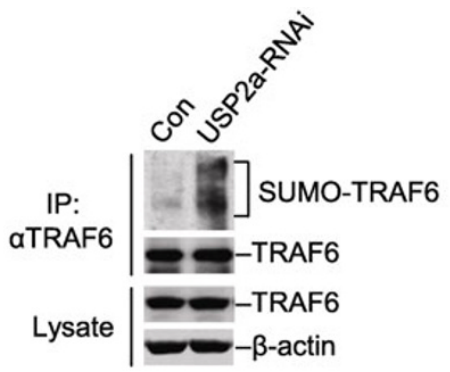

B

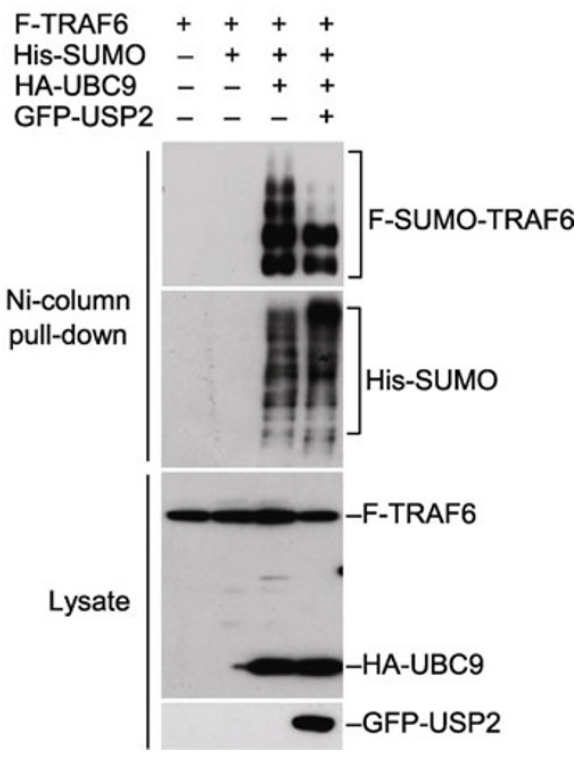

Figure 5. USP2a mediates deSUMOylation of TRAF6. (A) Jurkat $\mathrm{T}$ cells stably transfected with USP2a-RNAi or control RNAi were lyzed and immunoprecipitated and analyzed by immunoblots with the indicated antibodies $\left(6 \times 10^{6}\right.$ for each lane). (B) HEK293 cells $\left(2 \times 10^{6}\right)$ were transfected with the indicated plasmids (4 $\mu \mathrm{g}$ each). SUMOylation assays and immunoblot analysis were performed.

and Jentsch, 2009). Our findings that USP2a deSUMOylated TRAF 6 and promoted the association between MALT1 and TRAF6 suggest that SUMOylation of TRAF6 might prevent the TRAF6-MALT1 interaction.

Based on our observations, we propose a working model on the role of USP2a in TCR signaling. In this model, USP2a is constitutively associated with TRAF6 and keeps TRAF6 unSUMOylated under physiological conditions. Upon TCR stimulation, USP2a facilitates the interaction of unSUMOylated TRAF6 with MALT1. The recruitment of TRAF6 to the MALT1 complex is critical for activation of the E3 ubiquitin ligase activity of TRAF6, which then mediates the K63-linked polyubiquitination of MALT1 and itself, a step critical for signaling downstream NF-kB activation. This model can explain our observation that knockdown of USP2a inhibited K63-linked polyubiquitination of MALT1 and TRAF6. It is possible that knockdown of USP2a increases SUMOylation of TRAF6, blocks the recruitment of TRAF6 to MALT1 and subsequent activation of the E3 ligase activity of TRAF6, therefore, leads to inhibition of K63-linked polyubiquitination of MALT1 and TRAF6. Although more detailed mechanisms still need to be investigated, our study establishes an important role for USP2a in TCR-mediated signaling and provides an example on a possible role of an ubiquitin-related protease in deSUMOylation.

\section{MATERIALS AND METHODS}

\section{Reagents}

The antibodies against the following antigens were purchased from the indicated companies: antibodies against human USP2a (Abgent), CD3, CD28 and mouse IgG1 (BD Biosciences), Flag epitope and $\beta$-actin (Sigma), haemagglutinin (HA) epitope and ubiquitin (Origene), BCL10 (Santa Crus), human SUMO-1 (Zymed), human IKB $\alpha$ and phosphor-IkBa (Ser32/36) (Cell Signaling Technology) were purchased from the indicated companies. Mouse antisera were raised against recombinant human CARMA1, MALT1 and TRAF6 respectively. Human IL-2 ELISA kit (Boster), phorbol myristate acetate (PMA) (Promega), calcium ionophore lonomycin (Calbiochem), InterPlay Mammalian TAP system (Stratagene), His Pur Ni-NTA Resin (Thermo), TRIzol RNA isolation reagent (Invitrogen) and Gamma Bind G Plus-Sepharose (GE Healthcare Life Sciences) were purchased from the indicated companies.

\section{Plasmid constructs}

Mammalian expression plasmids for human CARMA1, BCL10, MALT1, TRAF6 or USP2a, fused with Flag- or HA- epitope, were constructed by standard molecular biology techniques. Sequences of RNAi constructs for knocking down the expression of human USP2a were previously described (He et al., 2012).

\section{Protein purification and mass spectrometry analysis}

HEK293 cells $\left(5 \times 10^{8}\right)$ stably transfected with pCTAP-MALT1 were collected. Cell lysates were subjected to tandem affinity purification procedures. The purified MALT1-associated proteins were digested by trypsin in solution, and the tryptic peptides were analyzed by HPLC-ESI/MS/MS with a Thermo Finnigan LTQ adapted for nanospray ionization. The tandem spectra were searched against Homo sapiens National Center for Biotechnology Information reference database using SEQUEST. Results were filtered by Xcorr+1 > 1.9, $+2>2.2,+3>3.5, \mathrm{sp}>500$, Deltcn $>0.1, \mathrm{Rsp} \leq 5$.

\section{Transfection}

HEK293 cells were transfected with mammalian expression plasmids by standard calcium phosphate precipitation. Jurkat $\mathrm{T}$ cells were transfected with siRNAs or expression plasmids by retroviral transduction (Li et al., 2012).

\section{Cell sorting by FACS}

Constructs of MIGR-GFP-USP2a were transfected into USP2aknockdown Jurkat $T$ cells. Forty-eight hours later, GFP-positive cells 
were isolated based on fluorescence intensity using the MoFlo XDP Sorter (Beckman Coulter). Approximately $1 \times 10^{5}$ cells of each sample with $>95 \%$ purity were obtained and cultured for further experiments.

\section{Coimmunoprecipitation and ubiquitination analysis}

For coimmunoprecipitation experiments, HEK293 cells or Jurkat T cells were lysed in NP-40 lysis buffer $(20 \mathrm{mmol} / \mathrm{L}$ Tris, $150 \mathrm{mmol} / \mathrm{L}$ $\mathrm{NaCl}, 1 \% \mathrm{NP}-40,1 \mathrm{mmol} / \mathrm{L}$ EDTA, $\mathrm{pH} 7.4)$ supplemented with proteases inhibitors $(10 \mu \mathrm{g} / \mathrm{mL}$ aprotinin, $10 \mu \mathrm{g} / \mathrm{mL}$ leupeptin and $1 \mathrm{mmol} / \mathrm{L}$ phenylmethylsulfonyl fluoride). For each sample, $0.4 \mathrm{~mL}$ aliquot of lysate was incubated with $0.5 \mu \mathrm{g}$ antibody and $25 \mu \mathrm{L}$ GammaBind $G$ Plus-Sepharose at $4^{\circ} \mathrm{C}$ for $4 \mathrm{~h}$. The beads were washed for three times with $1 \mathrm{~mL}$ lysis buffer containing $0.5 \mathrm{~mol} / \mathrm{L}$ $5 \mu \mathrm{g}$ antibody and $25 \mu \mathrm{L}$ GammaBind G Plus-Sepharose at $4^{\circ} \mathrm{C}$ for $4 \mathrm{~h}$. The bead $\mathrm{NaCl}$. For ubiquitination analysis, HEK293 cells or Jurkat T cells were suspended in $100 \mu \mathrm{L}$ NP-40 lysis buffer containing $1 \%$ SDS and boiled at $95^{\circ} \mathrm{C}$ for $10 \mathrm{~min}$. The boiled samples were then diluted with NP-40 lysis buffer to decrease the concentration of SDS to $0.1 \%$ and applied to normal Co-IP procedures.

\section{Quantitative real-time PCR}

Total RNA was extracted from Jurkat T cells $\left(5 \times 10^{6}\right)$ using TRIzol reagent and reverse-transcribed with M-MLV reverse transcriptase following the manufacturer' instructions. Quantitative Real-time PCR was performed using the SybrGreen PCR Supermix on the CFX96 real-time system (Bio-Rad). In the experiment, housekeeping gene $G A P D H$ was used as calibrators for normalization. Sequences of primers were as followed: GAPDH, 5'-GAGTCAACGGATTTGGTC GT-3' and 5'-GACAAGCTTCCCGTTCTCAG-3'; IL-2, 5'-AGAACTCA AACCTCTGGAGGAAG-3' and 5'-GCTGTCTCATCAGCATATTCAC AC-3.

\section{ELISA}

Jurkat T cells $\left(\sim 1 \times 10^{6}\right)$ were stimulated with PMA $(1 \mathrm{mmol} / \mathrm{L})$ plus lonomycin $(1 \mathrm{mmol} / \mathrm{L})$ for $15 \mathrm{~h}$. IL-2 production in the culture medium was measured using a human IL-2 ELISA kit.

\section{SUMOylation assays}

Cells were lyzed with lysis buffer $(6 \mathrm{~mol} / \mathrm{L}$ Guanidunium- $\mathrm{HCl}, 20 \mathrm{mmol} / \mathrm{L}$ imidazole, $5 \mathrm{mmol} / \mathrm{L} \beta-\mathrm{ME}, 10 \mathrm{mmol} / \mathrm{L}$ Tris, $100 \mathrm{mmol} / \mathrm{L}$ sodium phosphate buffer, $\mathrm{pH}$ 8.0). The cell lysates were incubated with Ni-NTA resin beads at $4^{\circ} \mathrm{C}$ overnight. The beads were serially washed with buffer $A$ (lysis buffer containing $0.1 \%$ Triton $X-100$ ), buffer $B(8 \mathrm{~mol} / \mathrm{L}$ urea, $5 \mathrm{mmol} / \mathrm{L} \beta-\mathrm{ME}, 0.1 \%$ Triton $\mathrm{X}-100,10 \mathrm{mmol} / \mathrm{L}$ Tris, $100 \mathrm{mmol} / \mathrm{L}$ sodium phosphate buffer, $\mathrm{pH}$ 8.0) and buffer C ( $8 \mathrm{~mol} / \mathrm{L}$ urea, $5 \mathrm{mmol} / \mathrm{L} \beta-\mathrm{ME}, 0.1 \%$ Triton $X-100,10 \mathrm{mmol} / \mathrm{L}$ Tris, $100 \mathrm{mmol} / \mathrm{L}$ sodium phosphate buffer, $\mathrm{pH} \mathrm{6.3).} \mathrm{The} \mathrm{beads} \mathrm{were}$ eluted with the elution buffer $(200 \mathrm{mmol} / \mathrm{L}$ imidazole, $5 \%$ SDS, $30 \%$ glycerol, $720 \mathrm{mmol} / \mathrm{L} \beta-\mathrm{ME}, 0.00025 \%$ bromophenol blue, $150 \mathrm{mmol} / \mathrm{L}$ Tris- $\mathrm{HCl}, \mathrm{pH}$ 6.7) for $20 \mathrm{~min}$ at room temperature. The eluted proteins were analyzed by immunoblots with the indicated antibodies.

\section{Reporter assays}

HEK293 cells $\left(1 \times 10^{5}\right)$ were seeded on 24-well dishes and trans- fected the following day by a standard calcium phosphate precipitation method. Empty control plasmid was added to ensure that each transfection receives the same amount of total DNA. To normalize for transfection efficiency, $0.05 \mu \mathrm{g}$ of $\mathrm{pRL}$-TK (Renilla luciferase) reporter plasmid was added to each transfection. Twenty hours after transfection, luciferase assays were performed using a dual-specific luciferase assay kit (Promega).

\section{ACKNOWLEDGMENTS}

We thank members of our laboratory for technical help and discussions. This work was supported by grants from the National Natural Science Foundation of China (Grant Nos. 30700417, 30921001 and 31170835) and the Ministry of Science and Technology of China (No. 2012CB910201).

\section{ABBREVIATIONS}

BCL10, B-cell CLL-lymphoma 10; CARMA1, caspase-recruitment domain membrane-associated guanylate kinase protein 1; DeSI-1, deSUMOylating isopeptidase 1; IL-2, interleukin-2; MALT1, mucosa-associated lymphoid tissue lymphoma translocation gene 1; SENP, sentrin-specific proteases; SUMO, small ubiquitin-like modifier; TAB2, TAK1-binding protein 2; TAK1, transforming growth factor $\beta$-activated kinase 1 ; TCR, T cell receptor; TRAF6, tumor necrosis factor receptor-associated factor 6 ; USP2a, ubiquitin-specific protease $2 \mathrm{a}$

\section{REFERENCES}

Alberola-lla, J., Takaki, S., Kerner, J.D., and Perlmutter, R.M. (1997). Differential signaling by lymphocyte antigen receptors. Annu Rev Immunol 15, 125-154.

Bergink, S., and Jentsch, S. (2009). Principles of ubiquitin and SUMO modifications in DNA repair. Nature 458, 461-467.

Coornaert, B., Baens, M., Heyninck, K., Bekaert, T., Haegman, M., Staal, J., Sun, L., Chen, Z.J., Marynen, P., and Beyaert, R. (2008). $\mathrm{T}$ cell antigen receptor stimulation induces MALT1 paracaspase-mediated cleavage of the NF-kappaB inhibitor A20. Nat Immunol 9, 263-271.

Deng, L., Wang, C., Spencer, E., Yang, L., Braun, A., You, J., Slaughter, C., Pickart, C., and Chen, Z.J. (2000). Activation of the IkappaB kinase complex by TRAF6 requires a dimeric ubiquitin-conjugating enzyme complex and a unique polyubiquitin chain. Cell 103, 351-361.

Gaide, O., Favier, B., Legler, D.F., Bonnet, D., Brissoni, B., Valitutti, S., Bron, C., Tschopp, J., and Thome, M. (2002). CARMA1 is a critical lipid raft-associated regulator of TCR-induced NF-kappa $B$ activation. Nat Immunol 3, 836-843.

Ghosh, S., May, M.J., and Kopp, E.B. (1998). NF-kappa B and Rel proteins: evolutionarily conserved mediators of immune responses. Annu Rev Immunol 16, 225-260.

Hannoun, Z., Greenhough, S., Jaffray, E., Hay, R.T., and Hay, D.C. (2010). Post-translational modification by SUMO. Toxicology 278 , 288-293.

He, X., Li, Y., Li, C., Liu, L.J., Zhang, X.D., Liu, Y., and Shu, H.B. (2012). USP2a negatively regulates IL-1beta- and virus-induced NF-kappa B activation by deubiquitinating TRAF6. J Mol Cell Biol. 
(In Press).

Li, Y., Chen, R., Zhou, Q., Xu, Z., Li, C., Wang, S., Mao, A., Zhang, X., He, W., and Shu, H.B. (2012). LSm14A is a processing body-associated sensor of viral nucleic acids that initiates cellular antiviral response in the early phase of viral infection. Proc Natl Acad Sci U S A 109, 11770-11775.

Liu, Y., Song, R., Gao, Y., Li, Y., Wang, S., Liu, H.Y., Wang, Y., Hu, Y.H., and Shu, H.B. (2012). Protein kinase C-delta negatively regulates $T$ cell receptor-induced NF-kappaB activation by inhibiting the assembly of CARMA1 signalosome. J Biol Chem 287, 20081-20087.

Mahul-Mellier, A.L., Pazarentzos, E., Datler, C., Iwasawa, R., AbuAli, G., Lin, B., and Grimm, S. (2012). De-ubiquitinating protease USP2a targets RIP1 and TRAF2 to mediate cell death by TNF. Cell Death Differ 19, 891-899.

Metzig, M., Nickles, D., Falschlehner, C., Lehmann-Koch, J., Straub, B.K., Roth, W., and Boutros, M. (2011). An RNAi screen identifies USP2 as a factor required for TNF-alpha-induced NF-kappaB signaling. Int J Cancer 129, 607-618.

Oeckinghaus, A., Wegener, E., Welteke, V., Ferch, U., Arslan, S.C., Ruland, J., Scheidereit, C., and Krappmann, D. (2007). Malt1 ubiquitination triggers NF-kappaB signaling upon T-cell activation. EMBO J 26, 4634-4645.

Oh, K.H., Yang, S.W., Park, J.M., Seol, J.H., lemura, S., Natsume, T., Murata, S., Tanaka, K., Jeon, Y.J., and Chung, C.H. (2011). Control of AIF-mediated cell death by antagonistic functions of CHIP ubiquitin E3 ligase and USP2 deubiquitinating enzyme. Cell Death Differ 18, 1326-1336.

Pham, L.V., Zhou, H.J., Lin-Lee, Y.C., Tamayo, A.T., Yoshimura, L.C., Fu, L., Darnay, B.G., and Ford, R.J. (2008). Nuclear tumor necrosis factor receptor-associated factor 6 in lymphoid cells negatively regulates c-Myb-mediated transactivation through small ubiquitinrelated modifier-1 modification. J Biol Chem 283, 5081-5089.

Pomerantz, J.L., Denny, E.M., and Baltimore, D. (2002). CARD11 mediates factor-specific activation of NF-kappaB by the $T$ cell receptor complex. EMBO J 21, 5184-5194.

Rebeaud, F., Hailfinger, S., Posevitz-Fejfar, A., Tapernoux, M.,
Moser, R., Rueda, D., Gaide, O., Guzzardi, M., lancu, E.M., Rufer, $\mathrm{N}$, et al. (2008). The proteolytic activity of the paracaspase MALT1 is key in T cell activation. Nat Immunol 9, 272-281.

Ruefli-Brasse, A.A., French, D.M., and Dixit, V.M. (2003). Regulation of NF-kappaB-dependent lymphocyte activation and development by paracaspase. Science 302, 1581-1584.

Ruland, J., Duncan, G.S., Elia, A., del Barco Barrantes, I., Nguyen, L., Plyte, S., Millar, D.G., Bouchard, D., Wakeham, A., Ohashi, P.S., et al. (2001). Bcl10 is a positive regulator of antigen receptor-induced activation of NF-kappaB and neural tube closure. Cell 104, 33-42.

Shan, J., Zhao, W., and Gu, W. (2009). Suppression of cancer cell growth by promoting cyclin D1 degradation. Mol Cell 36, 469-476.

Shin, E.J., Shin, H.M., Nam, E., Kim, W.S., Kim, J.H., Oh, B.H., and Yun, Y. (2012). DeSUMOylating isopeptidase: a second class of SUMO protease. EMBO Rep 13, 339-346.

Stevenson, L.F., Sparks, A., Allende-Vega, N., Xirodimas, D.P., Lane, D.P., and Saville, M.K. (2007). The deubiquitinating enzyme USP2a regulates the p53 pathway by targeting Mdm2. EMBO J 26, 976-986.

Sun, L., and Chen, Z.J. (2004). The novel functions of ubiquitination in signaling. Curr Opin Cell Biol 16, 119-126.

Sun, L., Deng, L., Ea, C.K., Xia, Z.P., and Chen, Z.J. (2004). The TRAF6 ubiquitin ligase and TAK1 kinase mediate IKK activation by BCL10 and MALT1 in T lymphocytes. Mol Cell 14, 289-301.

Thome, M., and Weil, R. (2007). Post-translational modifications regulate distinct functions of CARMA1 and BCL10. Trends Immunol 28, 281-288.

Wang, D., You, Y., Case, S.M., McAllister-Lucas, L.M., Wang, L., DiStefano, P.S., Nunez, G., Bertin, J., and Lin, X. (2002). A requirement for CARMA1 in TCR-induced NF-kappa B activation. Nat Immunol 3, 830-835.

Wegener, E., Oeckinghaus, A., Papadopoulou, N., Lavitas, L., Schmidt-Supprian, M., Ferch, U., Mak, T.W., Ruland, J., Heissmeyer, V., and Krappmann, D. (2006). Essential role for IkappaB kinase beta in remodeling Carma1-Bcl10-Malt1 complexes upon T cell activation. Mol Cell 23, 13-23. 